This item was submitted to Loughborough's Research Repository by the author.

Items in Figshare are protected by copyright, with all rights reserved, unless otherwise indicated.

\title{
Acquisition of fast transient signals in ICP-MS with enhanced time resolution
}

PLEASE CITE THE PUBLISHED VERSION

http://dx.doi.org/10.1039/C6JA00140H

PUBLISHER

(c) Royal Society of Chemistry

VERSION

AM (Accepted Manuscript)

\section{PUBLISHER STATEMENT}

This work is made available according to the conditions of the Creative Commons Attribution-NonCommercialNoDerivatives 4.0 International (CC BY-NC-ND 4.0) licence. Full details of this licence are available at: https://creativecommons.org/licenses/by-nc-nd/4.0/

\section{LICENCE}

CC BY-NC-ND 4.0

\section{REPOSITORY RECORD}

Managh, Amy, David N. Douglas, K. Makella Cowen, Helen Reid, and Barry L. Sharp. 2019. "Acquisition of Fast Transient Signals in ICP-MS with Enhanced Time Resolution". figshare.

https://hdl.handle.net/2134/21959. 
Received 00th January 20xx, Accepted 00th January 20xx

DOI: $10.1039 / \times 0 \times x 00000 x$

www.rsc.org/jaas

\title{
Acquisition of Fast Transient Signals in ICP-MS with Enhanced Time Resolution
}

\begin{abstract}
Amy J. Managh, ${ }^{a}$ David N. Douglas, ${ }^{a \dagger}$ K. Makella Cowen, ${ }^{a}$ Helen J. Reid, ${ }^{a}$ and Barry L. Sharp ${ }^{a}$
In recent years, the field of ICP-MS has seen an increasing trend towards sampling systems and methods that produce short transient signals, rather than a continuous signal response. Fast data acquisition, readout and storage are crucial to take advantage of the wealth of information available from these approaches. However, many of the current generation mass spectrometers, in particular sector-field instruments, were not designed to cope with such short duration signals. This article reports the use of a commercially available multi-channel scaler board, which facilitates capture of TTL pulses from an ICP-MS detector at a user defined time resolution down to $100 \mathrm{~ns}$. The board was used to profile $400-600 \mu \mathrm{s}$ wide signals with $10 \mu$ s resolution, derived from the nebulisation of a $50 \mathrm{~nm}$ gold nanoparticle suspension. Furthermore, the benefit of a $100 \%$ duty cycle was demonstrated for $10 \mathrm{~ms}$ wide signals, following ablation of individual macrophage cells with a fast response LA-ICP-MS interface.
\end{abstract}

\section{Introduction}

Inductively coupled plasma - mass spectrometry (ICP-MS) was first developed for the analysis of solutions, where a continuous flow of analyte is nebulised to produce a constant signal response. Although this form of analysis remains popular, alternative sampling approaches that produce a transient signal profile are now common. In recent years there has been a rise in single cell ${ }^{1-4}$ and single particle ${ }^{5,6}$ applications, where the analyte is diluted heavily before nebulisation, such that signals from individual particles or cells appear as single events. Several groups have reported the use of micro-droplet generators $(\mu D G)$, which deliver discrete droplets to the ICP-MS with up to $100 \%$ transport efficiency. ${ }^{7-9}$ Signals in the order of $500 \mu$ s duration have been reported for both single nanoparticles and cells using this approach., 9

Another notable form of transient sampling is laser ablation (LA), which is applied to sample solid targets, such as tissue sections $^{10-12}$ or isolated cells. ${ }^{13,}{ }^{14}$ LA-ICP-MS offers a combination of high sensitivity and high spatial resolution, which is critical to many bio-imaging applications. However, performing high resolution imaging within an acceptable time frame requires fast transit of the ablated material into the ICPMS instrument. As a result, a number of rapid response interfaces have been developed, which provide baseline separated signals in less than $10 \mathrm{~ms},{ }^{15-17}$ and are predicted to set the next paradigm in LA-ICP-MS technology. ${ }^{18,19}$

\footnotetext{
a Department of Chemistry, Loughborough University, LE11 3TU, UK

+ Present address: Inorganic Analysis, LGC Group, Teddington, London, TW11 OLY, UK.
}

For many years the software and hardware developed by instrument manufacturers did not evolve to meet the data acquisition requirements of the aforementioned fast transient applications. Thus, to improve the temporal resolution, some quadrupole users connected external hardware, such as oscilloscopes ${ }^{20,21}$ or custom built devices, ${ }^{22}$ to read the signal directly from the electron multiplier, or sacrificed sensitivity by opting for faster time-of-flight instrumentation. ${ }^{23,24}$ The latest product launches in quadrupole instrumentation reflect the developments in the field of particle analysis, with integration times of 10-100 $\mu$ s now available. However, strategies to improve acquisition time have not yet been widely reported for sector-field technology. This technical note addresses the issue through the use of a commercially available, "plug-andplay" multi-channel scalar board, which has a programmable time resolution, with a minimum bin width of 100 ns. Here, the capabilities of enhanced time resolution are demonstrated using a double focussing sector-field mass spectrometer (Element $\mathrm{XR}$ ), but the solution is equally applicable to other sector-field or quadrupole instruments. Two contemporary examples of fast-transient analysis are highlighted: single particle ICP-MS using a custom spray chamber, and the analysis of individual cells using a high speed LA-ICP-MS interface.

\section{Experimental}

Data Acquisition Instrumentation and Parameters. Analyses were performed using a sector-field ICP-MS (Element XR, Thermo Scientific, Bremen, Germany). A plug-in P7882 photon counter board (FAST ComTec GmbH, Oberhaching, Germany) was connected to the ion detection board of the Element $X R$ via a terminated $50 \Omega$ cable, in order to record the post 
electronic dead time corrected TTL pulse output. To prevent data losses, the detector saturation or 'trip' cable was disconnected from the ion detection board and ion beam deflection was disabled on the ESA board of the Element XR. ${ }^{\S}$ A trigger cable was used to synchronise data acquisition on the plug-in board with the start pulse from a laser ablation system. The ICP-MS was operated according to a previously reported fast-transient data acquisition protocol. ${ }^{25}$ In brief, this used low resolution mode, the E-scan setting, pulse counting detection mode, an integration time of $100 \mu \mathrm{s}, 1000$ integrations or "samples" per peak, and a mass window of $20 \%$. One isotope $\left({ }^{197} \mathrm{Au}\right)$ was analysed per run. Signals were simultaneously monitored using the MCDWIN software, supplied with the photon counter board, and the standard vendor software. The integration time on the board was set to $10 \mu \mathrm{s}$ and $100 \mu \mathrm{s}$ for the single particle and single cell analyses, respectively, with a maximum of 524288 integrations per run in both cases. Data was exported from the MCDWIN software in tab delimited format as .txt files, whilst the raw data points obtained from the vendor software were exported as .csv files. A custom Live Code based app (LA-ICP-MS Data Tool v0.17, Reid-IT Limited, Loughborough, UK) was used to identify and integrate individual peaks in both sets of files. The resulting peak information was exported to IGOR Pro 6.22A (Wavemetrics Inc., OR, USA) for graphical comparison.

Laser Ablation of Gold Labelled Cells. Human regulatory macrophages (Mregs) were generated and cultured with 50 $\mathrm{nm}$ gold nanoparticles (Sigma, Munich, Germany), as described previously. ${ }^{11}$ Following labelling, the Mregs were washed three times in Dulbecco's PBS and plated into chamber slides (BD Falcon, Heidelberg, Germany), where they were allowed to adhere for $24 \mathrm{~h}$. The average gold uptake was previously determined to be $3.39 \times 10^{8} \mathrm{Au}$ atoms/cell. ${ }^{11}$ Single cell sampling was performed using a UP-213 laser ablation system (Electro Scientific Industries, Huntingdon, UK), which was modified with a custom two-volume laser ablation cell and Dual Concentric Injector (DCl) inductively coupled plasma torch, described in detail elsewhere. ${ }^{16}$ The design used an unbroken, continuous diameter fused silica conduit, which extended from the point of ablation into the base of the plasma. The total path length was $c a .100 \mathrm{~cm}$. Helium was used as the ablation gas, at a flow rate of $0.1 \mathrm{l} / \mathrm{min}$, with an argon make-up gas introduced through the outer injector of the torch, at a rate of $1.4 \mathrm{l} / \mathrm{min}$. Ablation of individual Mregs was performed using a $55 \mu \mathrm{m}$ spot size and fluence of 6.0 $\mathrm{J} / \mathrm{cm}^{2}$, to ensure the entire cell was ablated. Only individual cells were targeted; cells present in clusters or spaced less than $55 \mu \mathrm{m}$ apart were not sampled.

Nebulisation of Gold Nanoparticles. A $50 \mathrm{~nm}$ gold nanoparticle suspension containing $\sim 3.5 \times 10^{10}$ particles $/ \mathrm{ml}$ in $0.1 \mathrm{mM}$ PBS (Sigma, Munich, Germany) was diluted in DI water (Milli-Q, Merck Millipore, Darmstadt, Germany) to yield a suspension containing $\sim 1 \times 10^{5}$ particles $/ \mathrm{ml}$. The solution was nebulised using a low flow rate nebuliser (EnyaMist, Burgener Research Inc., ON, Canada), which was fed by a syringe pump
(PHD Ultra, Harvard Apparatus, MA, USA) operated at a flow rate of $10 \mu \mathrm{l} / \mathrm{min}$. The nebuliser was supported inside a custom single pass spray chamber of $14 \mathrm{~mm}$ i.d. $\times 70 \mathrm{~mm}$ length (H. Baumbach \& Co. Ltd., Ipswich, UK) using an in-house 3D printed insert. Due to the large number of data points per file, the analysis was performed over series of 250 runs.

\section{Results and discussion}

Laser Ablation of Gold Labelled Cells. The ICP-MS was operated using a fast data acquisition method, which used the shortest permissible integration time of $100 \mu \mathrm{s}$, with 1000 integrations per peak, and a mass window of $20 \%$. This yielded a theoretical acquisition window of $20 \mathrm{~ms}$ (i.e. $200 \times 100 \mu \mathrm{s}$ ) in which to capture signal events. In practice, two timing anomalies were observed in the raw data files when using the vendor software. A number of integrations displayed background count rates of 9260,18520 or 27780 counts per second. Multiplying by the chosen integration time of $100 \mu \mathrm{s}$ therefore equates to $0.926,1.852,2.778$ counts. It is impossible to have a fraction of a count, so calculating the integration time back from the count rate (i.e. 1/9260) yields an actual integration time of closer to $108 \mu \mathrm{s}$. It was further noted that the raw data files contained 184 data channels per sweep, rather than the 200 ( $20 \%$ of the mass peak) that were set in the method. These observations were supported by the
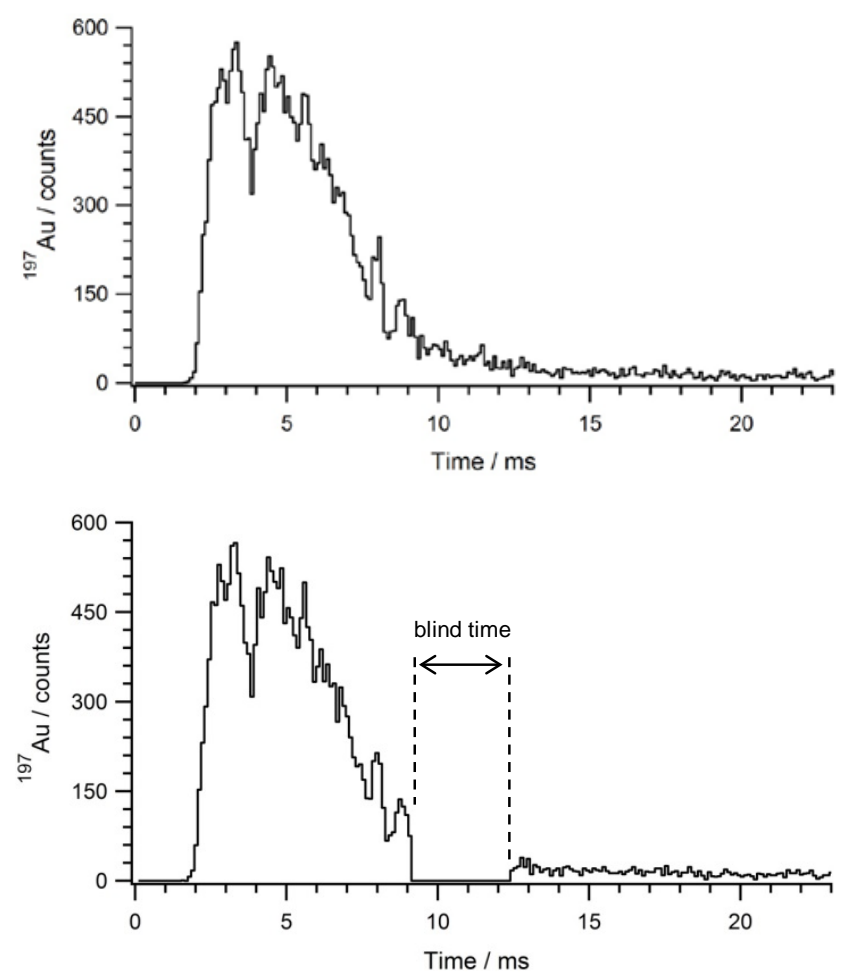

Fig 1. Example ${ }^{197} \mathrm{Au}$ signals for the LA-ICP-MS analysis of a single Au labelled macrophage. Top: signal from the plug-in data acquisition board. Bottom: corresponding output from the vendor software, adjusted to show data points at $108 \mu \mathrm{s}$ intervals. 
good alignment of the corrected time integrations with the data from the external board, as illustrated in Figure 1.

Figure 1 shows the comparison of a typical laser ablation signal recorded by the board and simultaneously by the vendor software. The observed peak duration of approximately $8 \mathrm{~ms}$ (full width at $10 \%$ maximum) is comparable to previous literature for biological analysis using the $\mathrm{DCl}$ interface. ${ }^{16}$ Following each sweep of the acquisition window, the ion beam is normally deflected onto the wall of the electrical sector, whilst the electrostatic analyser returns to the start of the window to begin the next scan, 'flyback'. This leads to a data loss or "blind time" of approximately $3 \mathrm{~ms}$ per sweep. For this experiment, ion beam deflection during flyback was disabled, hence the ions reached the detector during the re-setting period and there were no blind time losses in the data from the acquisition board. In contrast, the vendor hardware/software is not geared to allow retrieval of this information and a data loss of 3 ms duration was observed. It is clear that blind time disproportionately affects fast signals of this nature, where the data loss may account for a significant percentage of the peak, in comparison to those from more dispersive systems. The extent of the data loss is variable, depending on the position of the peak within the acquisition window, and is thus difficult to correct for during postprocessing. In this regard, the use of external devices to monitor $100 \%$ of the duty cycle may prove critical for quantitative imaging work using the next generation of fast response LA-ICP-MS interfaces.

Nebulisation of Gold Nanoparticles. Figure 2 depicts a signal from an individual nanoparticle event, simultaneously acquired using the plug-in board, at $10 \mu$ s resolution, and the vendor software, at $100 \mu \mathrm{s}$ resolution. In total, 995 nanoparticle events were recorded using the counter board. Typical pulse durations ranged between 400 and $600 \mu$ s at the baseline. The variance of the single particle integrated peak areas across the data set can be represented as a histogram (Figure 3 ), as is typical in particle sizing experiments. As a result of the blind time, 83 nanoparticle events present in the board data were not registered by the vendor software. A further 54 signals, $6 \%$ of those recorded, overlapped the region between the acquisition window and the blind time. These were therefore only partially captured. This is reflected in the distribution of these signals towards the lower end of the histogram in the vendor data, which ultimately resulted in a marginally broader distribution of the fitted function (FWHM of 1464 counts), compared to that of the board (FWHM of 1381 counts), and a lower average integrated signal per nanoparticle of 2011 counts versus 2092 counts for the board. This data loss therefore produces a distortion in the observed particle size distribution. Strenge et al. recently performed a similar comparison for quadrupole instrumentation, when examining the benefits of fast acquisition time $(5 \mu \mathrm{s})$ on a home built data board, versus vendor software at low time resolution $(0.5-10$ $\mathrm{ms}) .^{22}$ In that study, the occurrence of particle coincidences in the vendor data (two particles within the same integration
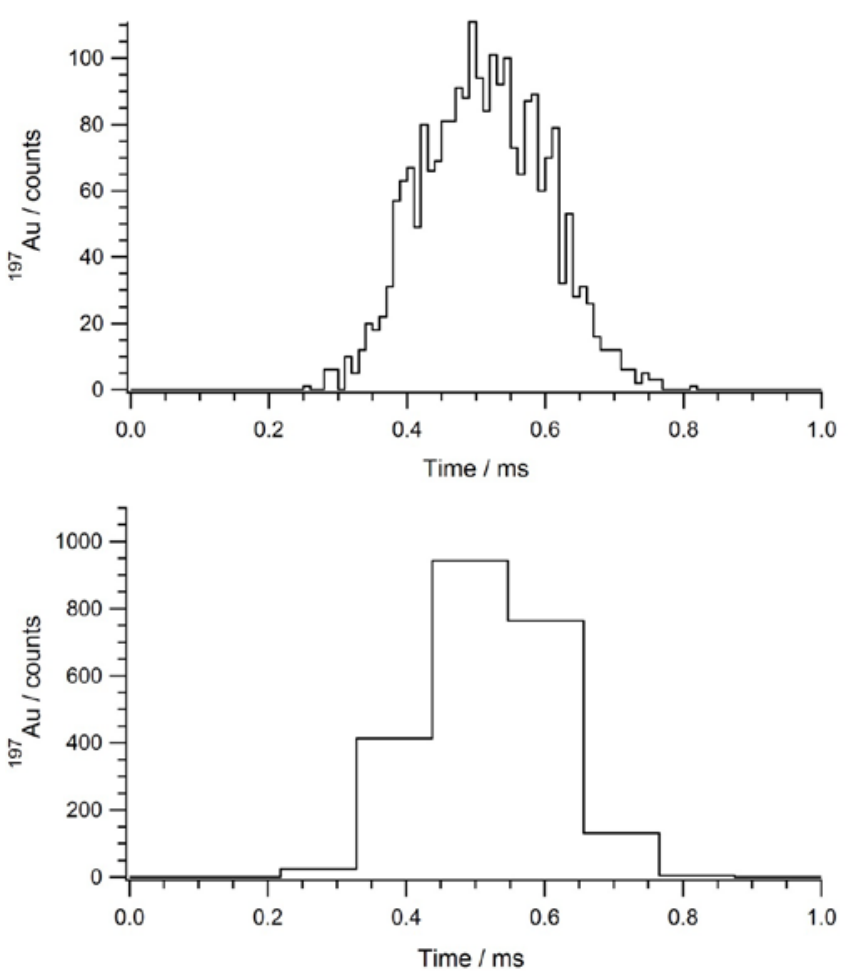

Fig 2. A typical ${ }^{197} \mathrm{Au}$ signal following nebulisation of a single 50 $\mathrm{nm}$ Au nanoparticle. Top: signal from the plug-in data acquisition board, with data points at $10 \mu \mathrm{s}$ intervals (integrated signal: 2276 counts). Bottom: output from the vendor software, with data points at $108 \mu$ s intervals (integrated signal: 2282 counts).

period) were a further contributing factor to the production of a wider distribution. These effects were not observed in the present study due to the heavy dilution factor, the slow sample introduction rate and the much lower integration time set in the Element software.

\section{Conclusions}

This technical note highlights the importance of data acquisition speed in fast transient ICP-MS analysis and presents a straightforward, "off-the-shelf" solution to improve the data acquisition characteristics of a mass spectrometer. In this manuscript the board was used in conjunction with a sector-field instrument (Thermo, Element XR), but the connections could be adapted to provide a universal solution to improve signal acquisition in both sector-field and quadrupole mass spectrometry. Use of the plug-in board provides significant advantages for fast transient work, including the removal of blind time in the duty cycle, which will become a significant factor as the next generation of laser ablation interfaces facilitate high speed imaging. The programmable nature of the board allows selection of 

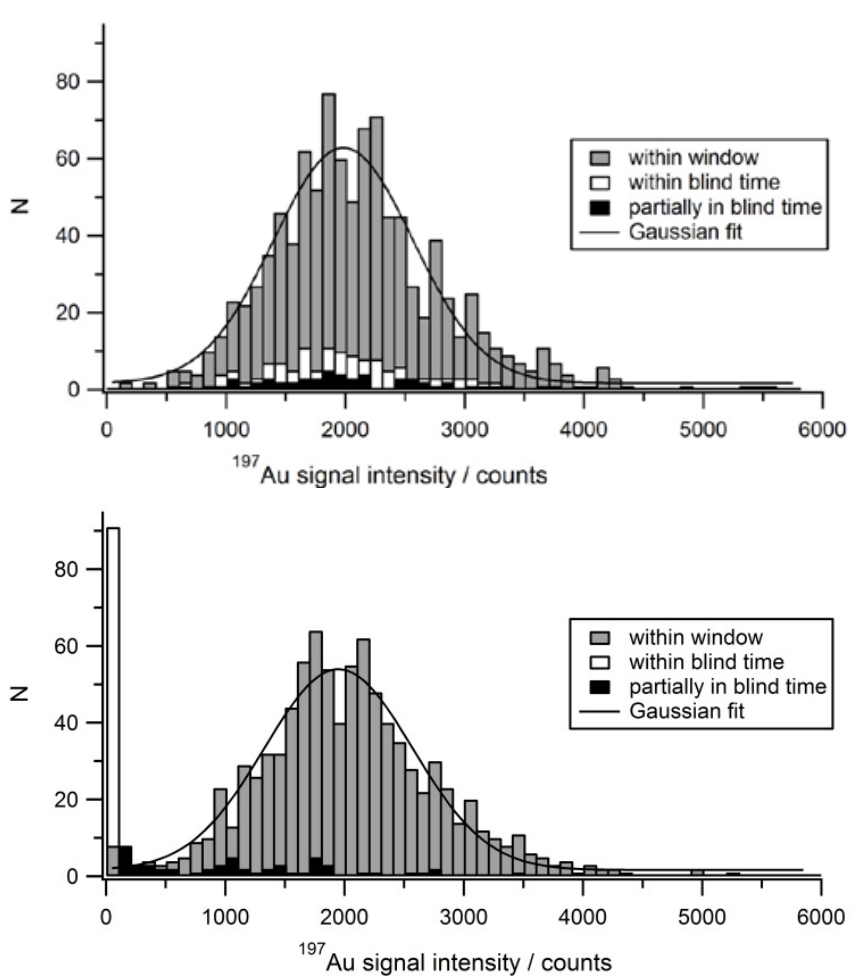

Fig 3. Stacked histograms showing the integrated ${ }^{197} \mathrm{Au}$ signals for 995 nanoparticle events observed using the plug-in data acquisition board (top) and the vendor software (bottom). Signals that occurred within the 'blind time' or across the border between the ICP measurement window and blind time were either missed or incompletely captured by the standard software, but were fully captured by the board.

integration times as low as 100 ns. At present, it would rarely be practical to operate down to this level in ICP-MS analysis, due to the low signal intensity and the limitation on the maximum number of integrations of 524288 per run with this board. In practice, a value of $100 \mu \mathrm{s}$, as used for the laser ablation section of this study, would be suited to many imaging applications. This would be sufficient for 5200 baseline separated data points when using a $<10 \mathrm{~ms}$ response time system, e.g. analysis of a $5.2 \mathrm{~mm}$ line at $1 \mu \mathrm{m}$ resolution, whilst retaining sufficient data points for examination of the detailed peak profile. The software takes approximately 3 seconds to save the data following every run, which can be easily automated using a script and carried out during the laser warm-up time for the next line. For nanoparticle analysis, where signals in the region of $500 \mu$ s are typical, a shorter integration setting of $10 \mu$ s revealed the fine signal structure. This level of detail is critical for the elimination of signal artefacts in particle sizing applications and may pave the way for fundamental studies into particle transport and plasma effects. Faster acquisition times may be also useful for multielemental analysis, where the user may wish to profile a single peak for multiple isotopes. However, the validity of the data in multi-element applications is also dependent of the scanning speed of the mass analyser, which remains a limiting factor.

\section{Acknowledgements}

This work was supported by the European Union Seventh Framework Programme ('The ONE Study', 260687) and the Engineering and Physical Sciences Research Council (Impact Acceleration Account). The authors wish to thank James Hutchinson and his colleagues at the University of Regensburg for preparation of the Au labelled macrophages, Lothar Rottman, Torsten Lindemann and David Hinds (Thermo Fisher Scientific) for their advice on connecting the data acquisition board to the Element XR, and Peter Reid (Reid-IT) for creation of a Live Code data processing app.

\section{Notes}

$\S$ The electrical connections discussed in this manuscript were implemented after consultation with Thermo Fisher Scientific. To minimise the risk of instrument damage or exposure to high voltages, users are advised to obtain advice from their instrument manufacturer before connecting external equipment to their mass spectrometers.

\section{References}

1. F. M. Li, D. W. Armstrong and R. S. Houk, Analytical Chemistry, 2005, 77, 1407-1413.

2. K. S. Ho and W. T. Chan, Journal of Analytical Atomic Spectrometry, 2010, 25, 1114-1122.

3. C. N. Tsang, K. S. Ho, H. Z. Sun and W. T. Chan, Journal of the American Chemical Society, 2011, 133, 7355-7357.

4. H. Wang, B. Wang, M. Wang, L. Zheng, H. Chen, Z. Chai, Y. Zhao and W. Feng, Analyst, 2015, 140, 523-531.

5. R. Peters, Z. Herrera-Rivera, A. Undas, M. van der Lee, H. Marvin, H. Bouwmeester and S. Weigel, Journal of Analytical Atomic Spectrometry, 2015, 30, 1274-1285.

6. W.-W. Lee and W.-T. Chan, Journal of Analytical Atomic Spectrometry, 2015, 30, 1245-1254.

7. S. Gschwind, L. Flamigni, J. Koch, O. Borovinskaya, S. Groh, K. Niemax and D. Gunther, Journal of Analytical Atomic Spectrometry, 2011, 26, 1166-1174.

8. B. Franze, I. Strenge and C. Engelhard, Journal of Analytical Atomic Spectrometry, 2012, 27, 1074-1083.

9. K. Shigeta, G. Köllensperger, E. Rampler, H. Traub, L. Rottmann, U. Panne, A. Okino and N. Jakubowski, Journal of Analytical Atomic Spectrometry, 2013, 28, 637-645.

10. J. O'Reilly, D. Douglas, J. Braybrook, P. W. So, E. Vergucht, J. Garrevoet, B. Vekemans, L. Vincze and H. Goenaga-Infante, Journal of Analytical Atomic Spectrometry, 2014, 29, 13781384.

11. A. J. Managh, R. W. Hutchinson, P. Riquelme, C. Broichhausen, A. K. Wege, U. Ritter, N. Ahrens, G. E. Koehl, L. Walter, C. Florian, H. J. Schlitt, H. J. Reid, E. K. Geissler, B. L. Sharp and J. A. Hutchinson, Journal of Immunology, 2014, 193, 2600-2608. 
12. D. N. Douglas, J. O'Reilly, C. O'Connor, B. L. Sharp and H. Goenaga-Infante, Journal of Analytical Atomic Spectrometry, 2016, 31, 270-279.

13. A. J. Managh, S. L. Edwards, A. Bushell, K. J. Wood, E. K. Geissler, J. A. Hutchinson, R. W. Hutchinson, H. J. Reid and B. L. Sharp, Analytical Chemistry, 2013, 85, 10627-10634.

14. D. Drescher, C. Giesen, H. Traub, U. Panne, J. Kneipp and N. Jakubowski, Analytical Chemistry, 2012, 84, 9684-9688.

15. S. J. M. Van Malderen, J. T. van Elteren and F. Vanhaecke, Journal of Analytical Atomic Spectrometry, 2015, 30, 119125.

16. D. N. Douglas, A. J. Managh, H. J. Reid and B. L. Sharp, Analytical Chemistry, 2015, 87, 11285-11294.

17. A. Gundlach-Graham, M. Burger, S. Allner, G. Schwarz, H. A. O. Wang, L. Gyr, D. Grolimund, B. Hattendorf and D. Günther, Analytical Chemistry, 2015, 87, 8250-8258.

18. S. J. M. Van Malderen, A. J. Managh, B. L. Sharp and F. Vanhaecke, Journal of Analytical Atomic Spectrometry, 2016, 31, 423-439.

19. A. Gundlach-Graham and D. Günther, Analytical and Bioanalytical Chemistry, 2016, 408, 2687-2695.

20. M. Tanner and D. Günther, Journal of Analytical Atomic Spectrometry, 2007, 22, 1189-1192.

21. S.-i. Miyashita, A. S. Groombridge, S.-i. Fujii, A. Minoda, A. Takatsu, A. Hioki, K. Chiba and K. Inagaki, Journal of Analytical Atomic Spectrometry, 2014, 29, 1598-1606.

22. I. Strenge and C. Engelhard, Journal of Analytical Atomic Spectrometry, 2016, 31, 135-144.

23. M. Tanner and D. Günther, Analytical and Bioanalytical Chemistry, 2008, 391, 1211-1220.

24. O. Borovinskaya, B. Hattendorf, M. Tanner, S. Gschwind and D. Günther, Journal of Analytical Atomic Spectrometry, 2013, 28, 226-233.

25. K. Shigeta, H. Traub, U. Panne, A. Okino, L. Rottmann and N. Jakubowski, Journal of Analytical Atomic Spectrometry, 2013, 28, 646-656. 
Table of contents entry

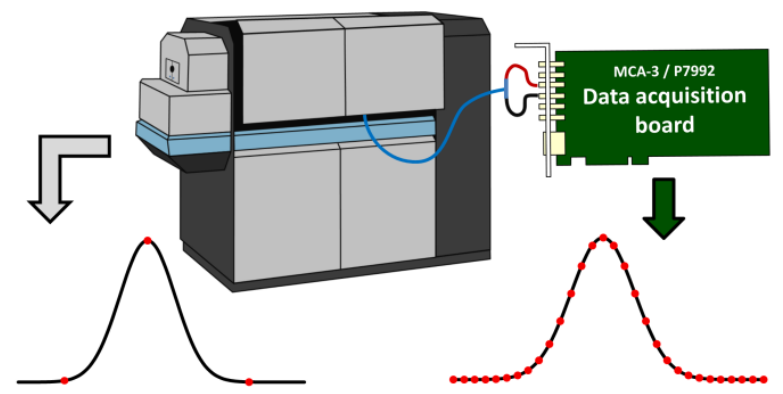

Novelty statement:

Improving time resolution in sector-field ICP-MS through use of a plug-in data acquisition board. 